\title{
Endovascular arch repair using the NEXUS arch endograft
}

\author{
Marina Ibrahim ${ }^{1}$, Thomas F. Lindsay ${ }^{2}$, Jennifer C. Y. Chung ${ }^{1}$, Kong T. Tan ${ }^{3}$, Juan Contreras ${ }^{1,4}$, \\ Maral Ouzounian ${ }^{1}$
}

\begin{abstract}
${ }^{1}$ Division of Cardiovascular Surgery, Peter Munk Cardiac Centre, Toronto General Hospital, University Health Network, University of Toronto, Toronto, ON, Canada; ${ }^{2}$ Division of Vascular Surgery Peter Munk Cardiac Centre, Toronto General Hospital, University Health Network, University of Toronto, Toronto, ON, Canada; ${ }^{3}$ Division of Interventional Radiology, Peter Munk Cardiac Centre, Toronto General Hospital, University Health Network, University of Toronto, Toronto, ON, Canada; ${ }^{4}$ Department of Surgery, Universidad De la Frontera, Temuco, Chile

Correspondence to: Maral Ouzounian, MD, PhD, FRCSC. Division of Cardiac Surgery, Toronto General Hospital, 200 Elizabeth St. 4N-464, Toronto M5G2C4, Canada. Email: Maral.ouzounian@uhn.ca.
\end{abstract}

Submitted May 16, 2021. Accepted for publication Aug 25, 2021.

doi: 10.21037/acs-2021-taes-18

View this article at: https://dx.doi.org/10.21037/acs-2021-taes-18

\section{Clinical vignette}

A 70-year-old female presented with asymptomatic aneurysmal degeneration of her arch and thoracoabdominal aorta in the setting of a chronic type B dissection. The maximal diameter of her proximal descending thoracic aorta (DTA) was $6.7 \mathrm{~cm}$. She was turned down for open thoracoabdominal aortic repair due to morbid obesity with poor functional capacity and limited mobility.

After multidisciplinary evaluation, we opted for staged endovascular repair with the NEXUS arch endograft [Herzlia, Israel] (1). Pre-operative arch debranching (2) was performed with carotid-carotid and left carotid-subclavian bypass to facilitate Zone 0 deployment (1).

\section{Surgical techniques}

NEXUS is a modular "off-the-shelf" device. It composes a main module for the arch and proximal descending aorta with an integrated brachiocephalic trunk (BCT) branch, and a proximal module for the ascending aorta. The two modules are connected through an interlocking latch mechanism.

\section{Preparation and vascular access}

The procedure was performed under general anesthesia. The right common femoral artery (CFA) and right midbrachial artery were exposed. A Perclose (Plymouth,
Minneapolis) device and an 8Fr sheath were inserted in the left CFA. A temporary pacing wire was advanced into the right ventricle.

\section{Cannulation of the BCT}

A catheter was advanced from the right brachial artery over a Glidewire (Terumo Medical, Tokyo, Japan) into the true lumen to the aortic bifurcation. This wire was snared through the right CFA providing through-and-through access. Heparin was administered for an ACT of $>300$ seconds. A Dav catheter (COOK Medical, Bloomington, Indiana) was introduced into the innominate artery and the right common carotid artery (RCCA) was cannulated with a V18 wire (Boston Scientific, Marlborough, Massachusetts, USA). A pigtail catheter was advanced into the ascending aorta.

\section{Thoracic stent graft deployment}

The right groin sheath was upgraded to a $22 \mathrm{Fr}$ Gore DrySeal sheath (Flagstaff, Arizona) and a Gore TAG reverse tapered thoracic stent graft (34-42-150 mm) was deployed in the proximal DTA. This was positioned first in order to expand the true lumen in the distal portion of the dissection.

\section{NEXUS arch stent graft deployment}

The NEXUS stent graft was advanced over the through- 
and-through wire into the arch, with the BCT branch in the innominate trunk. After confirming the position in multiple views, the BCT and arch stent graft were deployed with the distal graft portion in the Gore TAG stent graft. The BCT branch was post-dilated with a Coda balloon [COOK Medical] under rapid ventricular pacing. The through and through wire was pushed in from both ends to form a loop in the ascending aorta and subsequently advanced inside the left ventricle.

\section{NEXUS ascending aorta stent graft deployment}

The ascending module was advanced over the through and through wire into the ascending aorta. The ascending module was deployed under rapid ventricular pacing in Zone 0. The junction of the two components was post-dilated with a Coda balloon. Final angiography demonstrated filling of both coronaries and arch vessels. No evidence of retrograde type A aortic dissection (RTAD) or endoleak was noted. The patient awoke with normal neurological function.

\section{Post-operative outcome}

A post-operative computed tomography (CT) scan revealed an asymptomatic intramural hematoma (IMH) in her ascending aorta below the proximal end of the ascending graft. Given her comorbidities, conservative management with strict blood pressure control was implemented. Initial scans were stable, however, a three-month surveillance scan revealed evolution of the IMH into a frank type A dissection with ascending aortic expansion. She underwent urgent open repair with moderate hypothermia and antegrade cerebral perfusion. The ascending aorta module of the NEXUS graft was extracted, and her proximal aorta reconstructed. Recovery was uneventful. Four months later, the patient underwent successful endovascular thoracoabdominal aneurysm repair with a custom made four-vessel fenestrated stent-graft to complete the staged therapy for her chronic dissection. Follow-up scans revealed a type 3 endoleak at the distal arch graft with interval growth of the proximal DTA. She subsequently underwent relining of the distal part of the arch graft with a Gore TAG thoracic stent graft (40-40-150 mm). Her last intervention was a year later, where she underwent embolization of her inferior mesenteric artery and lumbar arteries for type 2 endoleaks.

\section{Comments}

Gold standard therapy for aortic arch repair is conventional surgery. This provides definitive repair for a wide variety of anatomical and pathological conditions; however, it is invasive and associated with significant morbidity. Endovascular arch repair is an attractive alternative in patients at high and prohibitive risk for open repair.

Endovascular arch repair technologies are rapidly evolving (3). Challenges seen in early series include elevated stroke rates, type $1 \mathrm{~A}$ endoleaks and RTAD. Our early experience with the NEXUS system in five patients has been promising (4). Three had saccular aneurysms of the arch, two had chronic dissections and all had significant comorbidities precluding open arch repair. Each patient underwent pre-procedural cervical debranching. There were no periprocedural deaths, strokes, or renal dysfunction. During follow-up, two patients required relining of the distal arch graft with an additional stent graft for type 3 endoleak.

Advantages of the NEXUS device include the integrated BCT branch that avoids branch separation, branch cannulation and the interlocking latch mechanism that avoids migration or modular separation. The NEXUS system is a single branch device requiring debranching of the supra-aortic vessels. This may reduce the risk of periprocedural cerebral embolization secondary to aortic arch manipulation. The through-and-through wire technique allows for secure, reliable tracking and reduces device manipulation during positioning and orientation. The device is not custom-made and is therefore accessible for urgent cases; however, anatomic limitations apply. Finally, the ascending aortic module of the stent graft maximizes available length of seal and minimizes the risk of type $1 \mathrm{~A}$ endoleak.

Despite these advantages, this case highlights important limitations. Early routine imaging is critical as RTAD may be asymptomatic. Index of suspicion should be high in those with a dilated ascending aorta and in the setting of aortic dissection (5). Ongoing imaging surveillance is mandatory as multiple re-interventions may be necessary.

Although conventional surgery remains standard for patients fit for open arch reconstruction, total endovascular repair is a valuable option for high-risk patients. Early results are promising, however larger and comparative studies are needed to determine safety and efficacy. 


\section{Acknowledgments}

MO is supported by the Antonio and Helga DeGasperis Chair in Clinical Trials and Outcomes Research at the University Health Network.

Funding: None.

\section{Footnote}

Conflicts of Interest: MO discloses a consulting agreement with Terumo, Cryolife, Edwards, and Medtronic. The other authors have no conflicts of interest to declare.

Open Access Statement: This is an Open Access article distributed in accordance with the Creative Commons Attribution-NonCommercial-NoDerivs 4.0 International License (CC BY-NC-ND 4.0), which permits the noncommercial replication and distribution of the article with the strict proviso that no changes or edits are made and the original work is properly cited (including links to both the formal publication through the relevant DOI and the license). See: https://creativecommons.org/licenses/by-nc-nd/4.0/.

Cite this article as: Ibrahim M, Lindsay TF, Chung JCY, Tan KT, Contreras J, Ouzounian M. Endovascular arch repair using the NEXUS arch endograft. Ann Cardiothorac Surg 2022;11(1):62-64. doi: 10.21037/acs-2021-taes-18

\section{References}

1. Mangialardi N, Serrao E, Ronchey S, et al. First Experience With the New Nexus Stent Graft for the Arch. J Vasc Surg 2015;61:94.

2. Konstantinou N, Debus ES, Vermeulen CFW, et al. Cervical Debranching in the Endovascular Era: A Single Centre Experience. Eur J Vasc Endovasc Surg 2019;58:34-40.

3. Chung JC, Ouzounian M, Chu MWA, et al. The Evolving Role of Hybrid Arch Repair. Innovations (Phila) 2020;15:506-12.

4. Planer D, Elbaz-Greener G, Mangialardi N, et al. NEXUS Arch: A Multicenter Study Evaluating the Initial Experience with a Novel Aortic Arch Stent Graft System. Ann Surg 2021. [Epub ahead of print]. doi: 10.1097/ SLA.0000000000004843.

5. Eggebrecht H, Thompson M, Rousseau H, et al. Retrograde ascending aortic dissection during or after thoracic aortic stent graft placement: insight from the European registry on endovascular aortic repair complications. Circulation 2009;120:S276-81. 